\section{The cost of living}

\section{Peter Rich}

$\mathrm{T}$ he 1960s was an important time for mitochondrial research, which saw many significant and enduring advances. During this period, Peter Mitchell developed the chemiosmotic proposal - the coupling of biological electron transfer to ATP synthesis. Although it ultimately won Mitchell a Nobel Prize, his work was highly controversial, and it was more than a decade before his ideas were accepted widely, leading some researchers to claim prior ownership of key elements of the ideas that still persist today.

The fact that mitochondria are central to the efficient provision of energy for eukaryotic cells is undisputed. It is generally accepted that the early and energetically inefficient eukaryotic cell was invaded more than a billion years ago by a bacterium containing a much more efficient system for utilizing available energy sources - the oxygenconsuming respiratory chain. The majority of the bacterial genetic information was subsequently transferred to the nucleus, transforming the bacterial symbionts into slave mitochondrial organelles.

We rely on these mitochondria to synthesize ATP. The process begins with the passage of electrons derived from food along a series of mitochondrial respiratory-chain carriers, until they are consumed in converting the oxygen we breathe into water. Mitchell showed that their transfer is linked to the movement of protons across the ion-impermeable inner

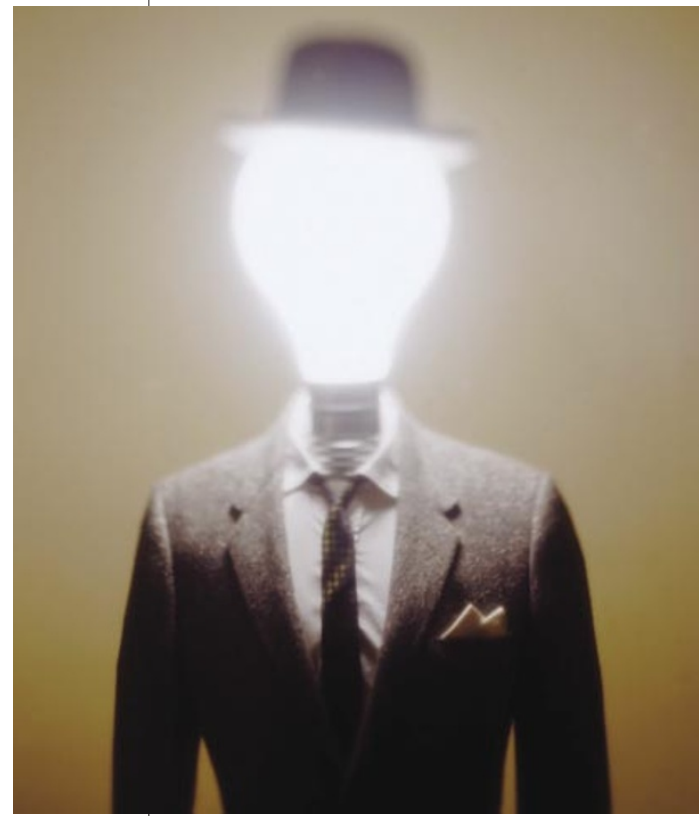

Shining example: even at rest, a human body requires as much power as a 100 -watt lightbulb. mitochondrial membrane in which the respiratory chains are embedded. The membrane acts as a capacitor, storing energy as a $\mathrm{pH}$ and charge difference across the membrane. This gradient of electrochemical potential energy is used to drive protons through the ATP synthases that are embedded in the same membrane, and which couple the exergonic proton flow to the synthesis of ATP from ADP and phosphate. The energy thus stored can be released by ATP hydrolysis, a reaction that is used by the myriad energy-requiring enzymes that maintain cellular function.

An average human at rest has a power requirement of roughly 100 kilocalories (420 kilojoules) per hour, which is equivalent to a power requirement of 116 watts - slightly more than that of a standard household lightbulb. But, from a biochemical point of view, this requirement places a staggering power demand on our mitochondria. Mitchell's work showed that the electrochemical gradient of protons across the inner mitochondrial membrane that drives ATP synthesis is roughly $200 \mathrm{mV}$, and most of this is the electric field component.

If it is assumed that $90 \%$ of human power is provided by the protons that are transferred through the ATP synthase, then the transmembrane proton flux would have to represent a current of $522 \mathrm{amps}$, or roughly $3 \times 10^{21}$ protons per second. Recent estimates, based on the structure of yeast ATP synthase, contend that three ATP molecules are formed for every ten protons that are transferred. Assuming a conversion efficiency that is close to unity, ATP is reformed at a rate of around $9 \times 10^{20}$ molecules per second, equivalent to a turnover rate of ATP of $65 \mathrm{~kg}$ per day and with much higher rates than this during periods of activity. This output is itself powered by the oxygen-consuming respiratory chain.

A typical adult male consumes around 380 litres of oxygen each day, and top athletes can sustain rates that are ten times greater for limited periods. Most (90\%) of this oxygen is reduced to water by the terminal respiratorychain enzyme, cytochrome oxidase. The inner mitochondrial membrane contains around 0.4 nanomoles of this enzyme per milligram of protein. It can work at a rate in excess of 300 electrons every second, but probably operates at an average rate of no more than 50 per second. Hence, an average human will need $2 \times 10^{19}$ molecules of cytochrome oxidase to support oxygen consumption. With the inner mitochondrial membrane having a lipid/protein weight ratio of $1: 1$, the cytochrome oxidase would be associated with about $70 \mathrm{ml}$ of lipoprotein membrane. However, the membrane's thickness - only $6 \mathrm{~nm}$ - means that the surface area of the inner mitochondrial

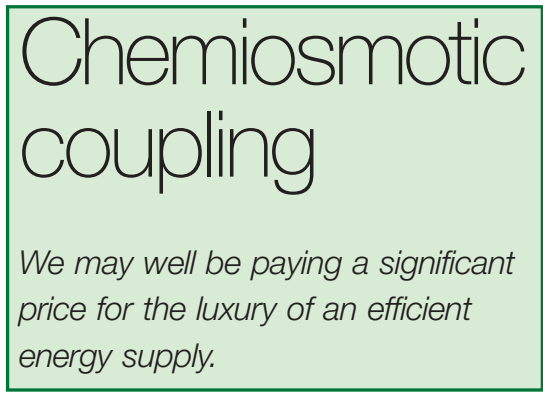

membrane in an average human would be around $14,000 \mathrm{~m}^{2}$.

This constant energy provision is a herculean task so it is not surprising that defects in mitochondrial function should lead to physiological disorders. As human mitochondrial DNA (mtDNA) sequence and clinical databases are amassed and compared, the number of diseases that are suspected to be caused by mitochondrial dysfunction due to mtDNA mutations is increasing. Mutations in mtDNA might even accumulate with age and contribute to a reduced efficiency of energy provision. Some aspects of mitochondrial function seem to be potentially deleterious to cell health, particularly the 'leakage' of electrons that must inevitably occur, resulting in the formation of small amounts of damaging and mutagenic free radicals. Mitochondria may also play a key role in some cells in an apoptotic, or 'cell death', pathway by releasing factors from the intermembrane space into the cytoplasm, where they interact with the caspase cascade system. So it seems that our energy-producing organelles have not yet been entirely tamed to the needs of the eukaryotic cells that control them, and that we may well be paying a significant price for the luxury of the efficient energy supply that we need to sustain our ravenous needs.

Recognition of such mitochondrial activities, together with the tremendous advances in determining the structural details of the mitochondrial molecular machinery, in many instances down to the atomic level, is heralding a new era of interest in mitochondria. Hopefully, the new wave of insights into this enigmatic organelle that are now emerging will not evoke the same controversy and prolonged difficulties in acceptance as Mitchell's work.

Peter Rich is in the Department of Biology, University College London, Gower Street,

London WC1E 6BT, UK.

\section{FURTHER READING}

Gilbert, G. N. \& Mulkay, M. Opening Pandora's Box (Cambridge Univ. Press, 1984).

Prebble, J. \& Weber, B. Wandering in the Gardens of the Mind: Peter Mitchell and the Making of Glynn (0xford Univ. Press, New York, 2003).

Catalogue of the Papers and Correspondence of Peter Dennis Mitchell FRS NCUAS (Cambridge Univ. Libr., Cambridge, UK, 1997).

Kiberstis, P. A. Science 283, 1475 (1999). 